\title{
Cervical Epidural Abscess Mimicking as Stroke - Report of Two Cases
}

\author{
Jagan Mohana Reddy Velpula ${ }^{*}$, Harinder Gakhar, Kohilavani Sigamoney and \\ Rajendra Bommireddy
}

Department of Orthopaedics, Royal Derby Hospital, Derby, UK

\begin{abstract}
Background: Stroke is a common provisional diagnosis in patients presenting to the emergency department (ED) with unilateral neurological deficit. Cervical epidural abscess (CEA) may also present clinically with a unilateral neurological deficit.
\end{abstract}

Objective: To highlight the inherent problems with diagnosing cervical epidural abscess and possible consequences of delay in diagnosis.

Case Report: We would like to highlight two cases provisionally diagnosed as stroke. Both cases turned out to be cervical epidural abscesses. The delay in diagnosis and treatment led to suboptimal outcome in both cases.

Summary: Cases with suspected stroke who deteriorate while under treatment or whose diagnosis is doubtful should have MRI whole spine in order to avoid potential complications.

Keywords: Discitis, stroke, cervical epidural abscess.

\section{INTRODUCTION}

Stroke is usually suspected when patients present with unilateral neurological deficit to the ED. In rare cases cervical discitis/ cervical epidural abscess may have a similar presentation. Cervical epidural abscesses are known for wrong or delay in diagnosis. We would like to highlight two cases provisionally diagnosed as stroke which turned out to be cervical epidural abscesses. The delay in diagnosis and treatment led to suboptimal outcome in both cases.

\section{Case 1}

67-year male presented to ED with a history of left arm and leg weakness of one-day duration. He reported to have fallen 4 times in the day. He had no significant past medical problems. Vital signs were unremarkable. Physical examination revealed MRC grade III/V power in all myotomes of his left upper and lower limb. There was no sensory loss. After initial assessment he was suspected to have a right middle cerebral artery territory stroke. He had routine bloods and also underwent a CT scan of his brain, which was normal. He clinically deteriorated over the next 48 hours with the weakness spreading to the right half of his body and requiring ventilatory support. He was then thought to have Landry Gullian-Barre Syndrome. His routine bloods showed white cell count of $14460 / \mathrm{mm} 3$ and C-reactive protein levels of $143 \mathrm{mg} / \mathrm{L}$. In keeping with the new provisional diagnosis he underwent a spinal tap that was normal. A subsequent MRI scan of the spine showed C6/7 discitis and extensive cervical epidural abscess extending

*Address correspondence to this author at the Department of Orthopaedics, Royal Derby Hospital, Derby, UK; Tel: 0044 7877461862;

E-mail: orthojagan@gmail.com from foramen magnum to T4. (Fig. (1): Case 1 T2W saggital sections showing C6/7 discitis and epidural abscess causing cord compression. Also shown is the post-op scan showing resolution of abscess but residual changes of spinal cord myelomalacia opposite C2-Fig. (2). He underwent urgent anterior cervical decompression and evacuation of pus. In spite of adequate decompression and antibiotic treatment and prolonged rehabilitation attempts at a spinal cord injury centre, neurological recovery was not adequate for self-care and ambulation.

\section{Case 2}

74-year female presented to casualty for being unwell for 2 weeks. She also complained of right side arm and leg weakness. In the past she did suffer from chronic obstructive pulmonary disease. On physical examination she had MRC grade III power in her right upper limb myotomes and MRC grade IV power in her right lower limb myotomes. She also had some sensory dulling but that was non dermatomal. She was provisionally diagnosed to have a left middle cerebral artery territory stroke. Her vital signs were normal. Her leukocyte count was raised $(13000 / \mathrm{mm} 3)$ and urine dip showed nitrates and leucocytes. A CT scan of her brain was performed, which was normal. Over the next day her weakness progressed to all four limbs. Next day she underwent a carotid Doppler scan which revealed a large pre-vertebral swelling. Subsequent MRI scan of her spine showed C4/5 discitis with a cervical epidural abscess causing cord compression which was decompressed surgically the same day. (Fig. (3): Case 2 Saggital T2W and Fig. (4): T1W pre-op images showing epidural abscess and cord compression). She had anterior decompression and culture sensitive antibiotic treatment. She recovered partially to regain MRC $3+/ 5$ motor power in her upper limbs. After one 


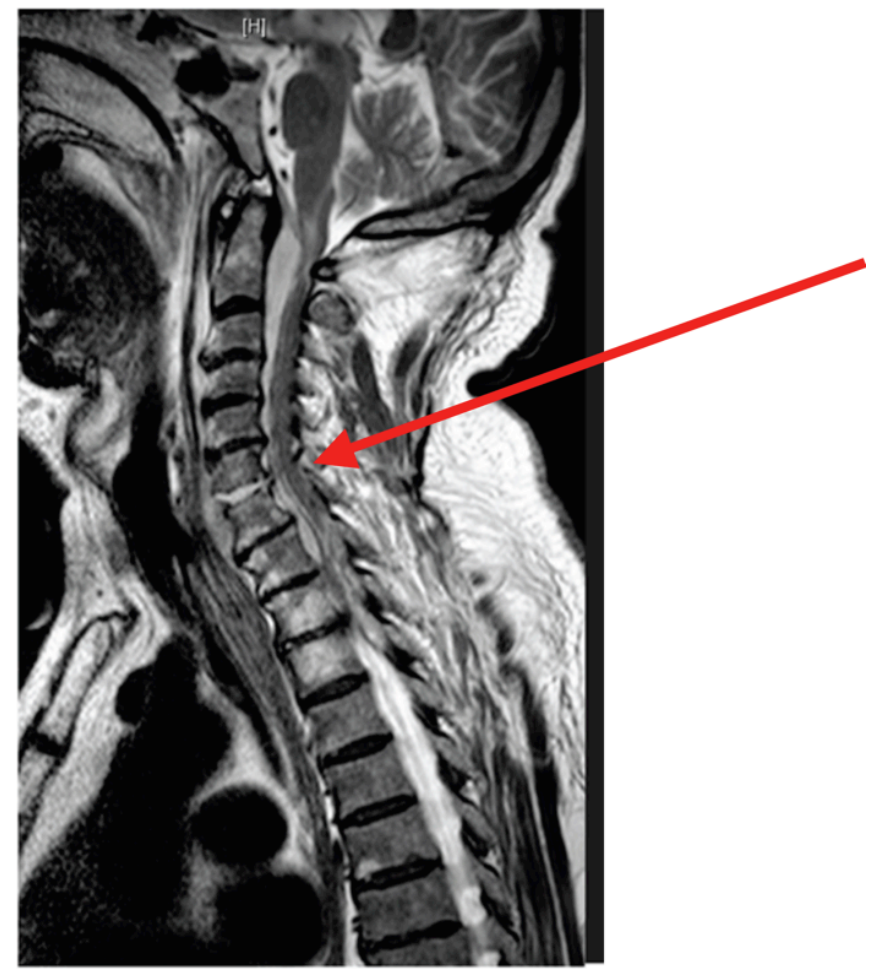

Fig. (1). Case $1 \mathrm{~T} 2 \mathrm{~W}$ saggital sections showing C67 discitis and epidural abscess causing cord compression.

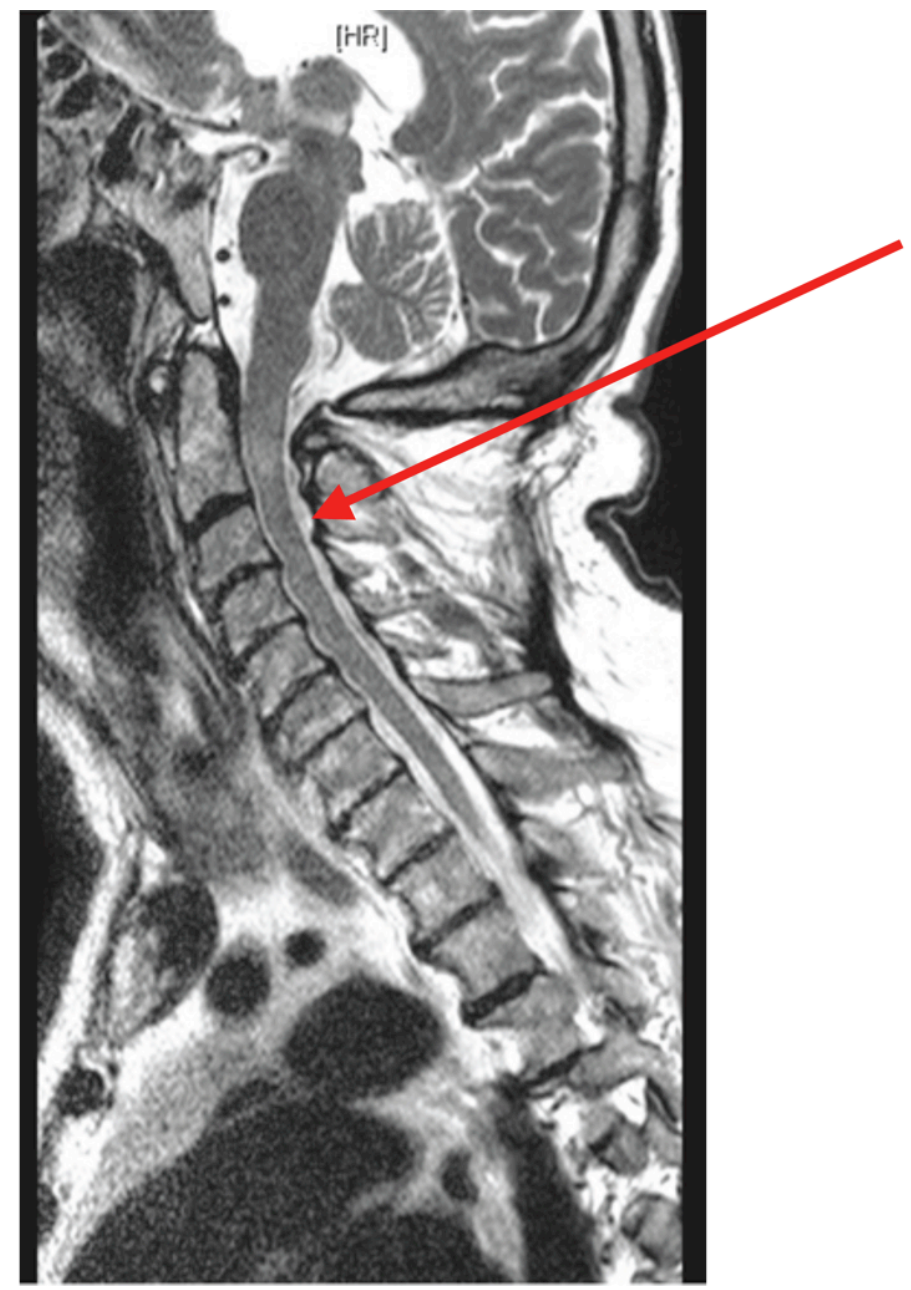

Fig. (2). post-op scan showing resolution of abscess but residual changes of spinal cord myelomalacia opposite C2. 


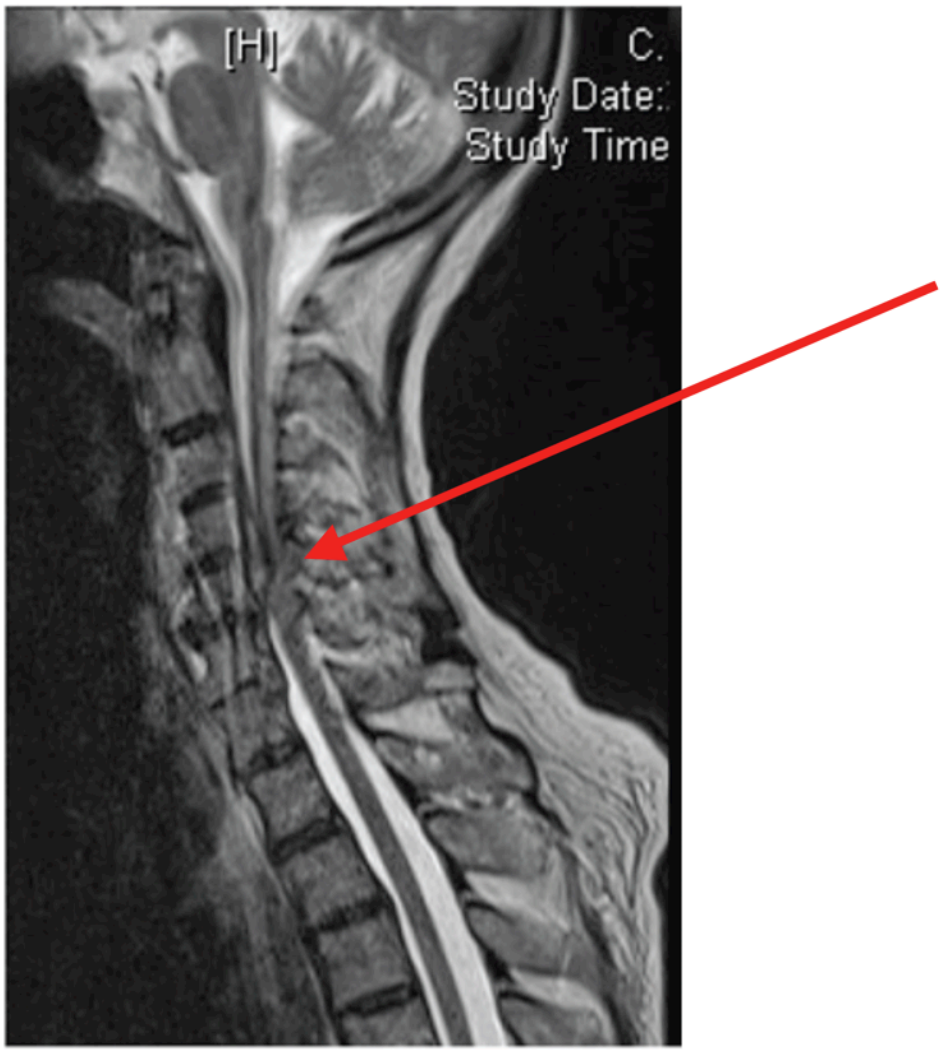

Fig. (3). Case 2 Saggital T2W pre-op images showing epidural abscess and cord compression.

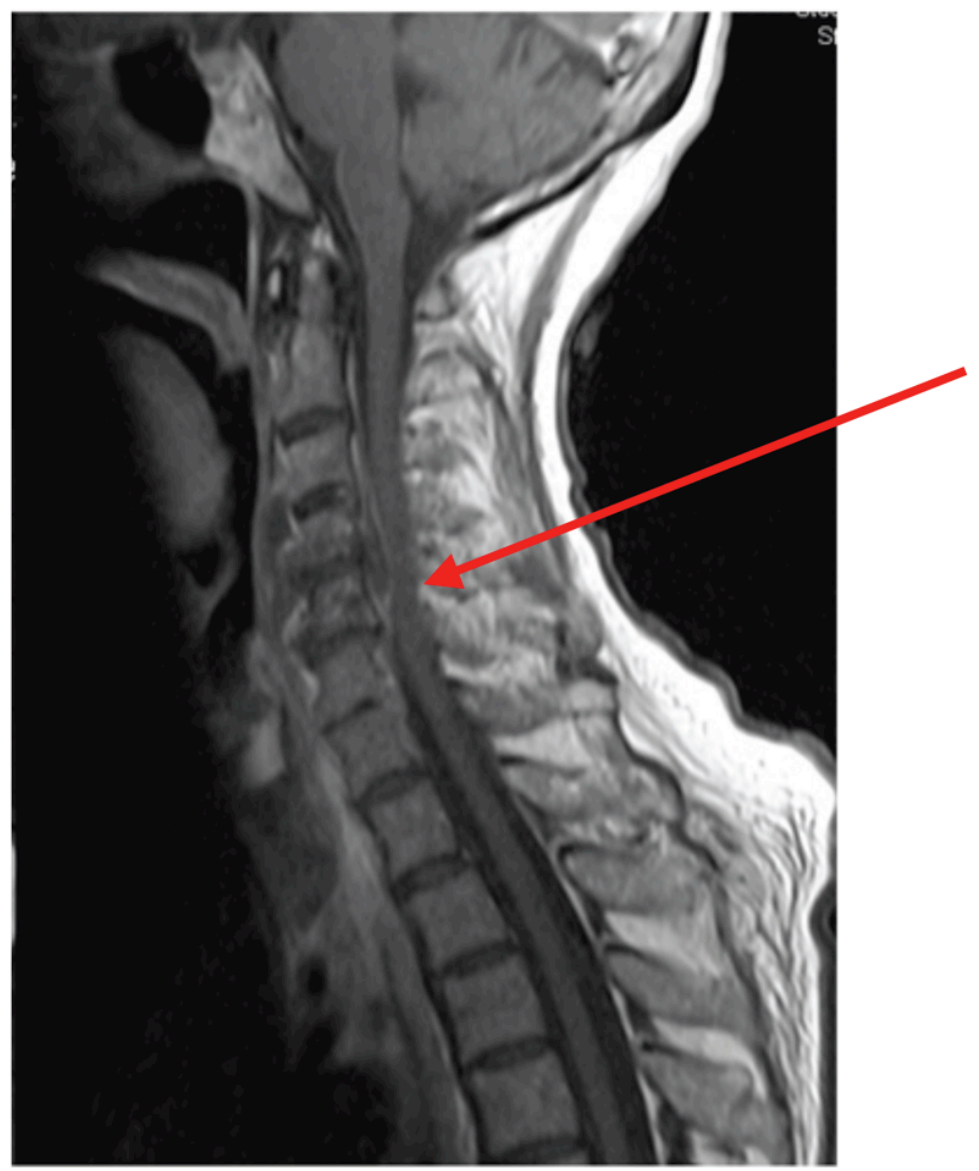

Fig. (4). Case 2 Saggital T1W pre-op images showing epidural abscess and cord. 
week she developed severe aspiration pneumonia requiring ventilatory support and passed away due to respiratory failure.

\section{DISCUSSION}

Stroke is the leading cause of serious long-term disability. According to the World Health Organisation, 15 million new cases of stroke occur every year of which 5 million die and 5 million are left disabled [1].

Cervical discitis and cervical epidural abscess is a true spinal emergency and potentially a life threatening disease. Cervical discitis and cervical epidural abscess is extremely rare and remain a diagnostic challenge $[2,3]$. Incidence of Cervical epidural abscess may be as low as 1 in 70,000 to 1 in 400,000 hospital admissions [4]. Multiple emergency department visits and diagnostic delays occur in $68 \%$ of patients, contributing to a $45 \%$ morbidity rate and $15 \%$ mortality $[5,6]$. Late recognition often leads to permanent weakness or paralysis.

Cervical epidural abscess/discitis may present with pain and/or fever and/or neurological deficit, in which case it is more likely to be suspected. In most cases the presentation is subtle and even then it may be overlooked because neck/back pain and fever are common presenting complaints in the emergency department. Prevalence of neck pain is high among the general population (approximately 15\%) [7]. The cases we have discussed here illustrate the need to suspect discitis or epidural abscess in cases which are either deteriorating under treatment or have not been fully diagnosed. Early diagnosis will help prevent serious deficits and disability.

\section{CONCLUSIONS}

Cervical discitis and epidural abscess should be considered as a differential diagnosis in suspected stroke. Patients with suspected stroke with negative CT brain scans, who deteriorate further, should be investigated. We suggest performing urgent MRI spine particularly in the presence of progressive deterioration that is outside the purview of a simple stroke.

\section{CONFLICT OF INTEREST}

The authors confirm that this article content has no conflict of interest.

\section{ACKNOWLEDGEMENTS}

Declared none.

\section{REFERENCES}

[1] The World Health Report. Reducing risks, promoting healthy life 2002.

[2] Darouiche RO. Spinal epidural abscess. N Engl J Med 2006; 355 : 2012-20.

[3] Mackenzie AR, Laing RB, Smith CC, Kaar GF, Smith FW. Spinal Epidural Abscess: The importance of early diagnosis and treatment J Neurol Neurosurg Psychiat 1998; 65: 209-12.

[4] Lasker BR, Harter DH. Cervical Epidural Abscess. Neurology 1987; 37: 1747-53

[5] Reihsaus E, Waldbaur H, Seeling W. Spinal epidural abscess: a meta-analysis of 915 patients. Neurosurg Rev 2000; 232: 175-204.

[6] Soehle M, Wallenfang T. Spinal epidural abscess: clinical manifestations, prognostic factors and outcomes. Neurosurgery 2002; 51(1): 79-87.

[7] Voorhies RM. Cervical spondylosis: recognition, differential diagnosis, and management. Ochsner J 2001; 3(2): 78-84. 